\title{
Prognostic significance of platelet-to-lymphocyte ratio in non-small-cell lung cancer: a meta-analysis
}

This article was published in the following Dove Press journal:

OncoTargets and Therapy

22 February 2016

Number of times this article has been viewed

\section{Guangliang Qiang \\ Chaoyang Liang \\ Fei Xiao \\ Qiduo Yu \\ Huanshun Wen \\ Zhiyi Song \\ Yanchu Tian \\ Bin Shi \\ Yongqing Guo \\ Deruo Liu}

Department of Thoracic Surgery, China-Japan Friendship Hospital, Beijing, People's Republic of China
Correspondence: Chaoyang Liang Department of Thoracic Surgery, ChinaJapan Friendship Hospital, Yinghua East Road No 2, Chaoyang District, Beijing 100029, People's Republic of China

Tel +8618610570066

Fax +861084206186

Email chaoyangliang8@yahoo.com
Background: The platelet-to-lymphocyte ratio (PLR) is a useful predictive factor in several cancers. However, the prognostic value of PLR in patients with non-small-cell lung cancer (NSCLC) is still indistinct. Therefore, it was necessary for us to perform a meta-analysis to assess the prognostic value of PLR in patients with NSCLC.

Methods: A systematic literature search was performed by using PubMed, EMBASE, and Web of Science databases for relevant studies until May 2015. Published studies investigating the association between PLR and overall survival (OS) and disease-free survival (DFS) were selected. Data from each eligible study were extracted. A meta-analysis was performed to analyze the prognostic value of PLR by using the hazard ratio (HR) and $95 \%$ confidence intervals (95\% CI).

Results: A total of seven studies involving 1,554 patients were included in our meta-analysis. Our pooled results demonstrated that high PLR was associated with poor OS (HR: 1.60, 95\% CI: $1.34-1.90, I^{2}=22.3 \%, P_{\text {heterogeneity }}=0.259$ ) and DFS (HR: $1.38,95 \%$ CI: $1.11-1.73, I^{2}=0 \%$, $P_{\text {heterogeneity }}=0.482$ ). Subgroup analysis between PLR and OS was performed in a further investigation. When the patients were segregated according to ethnicity, sample size, cutoff value, stage, and treatment modality, high PLR was also significantly correlated with OS. There was no significant heterogeneity among included studies.

Conclusion: High PLR is associated with poor prognosis in patients with NSCLC. PLR may be a significant predictive biomarker in patients with NSCLC.

Keywords: non-small cell lung cancer, platelet-to-lymphocyte ratio, prognosis, meta-analysis, overall survival, disease-free survival

\section{Introduction}

Lung cancer is the most common cause of cancer-related deaths with a 5-year survival rate of about $16 \% .{ }^{1}$ Non-small-cell lung cancer (NSCLC) accounts for approximately $80 \%$ of lung cancer diagnoses. Although there is a significant improvement in the multidisciplinary treatment of patients with NSCLC, the prognosis still remains poor. A promising breakthrough to improve the long-term survival is the application of accurate predictive markers that can guide therapeutic strategies and monitor disease progress. Therefore, it is crucial for us to identify better predictors for prognosis in patients with NSCLC.

In an attempt to better estimate the prognosis, many prognostic parameters have been investigated, such as tumor-node-metastasis (TNM) stage, performance status, weight loss, and other miscellaneous factors. Recently, it is widely recognized that systemic inflammatory response plays an important role in the initiation and progression of cancer. ${ }^{2}$ Molecular factors and biological pathways including upregulation of cytokines, chemokines and inflammatory mediators, promotion of angiogenesis, local immunosuppression, inhibition of apoptosis, and DNA damage are involved 
in this response and are associated with an increased risk of metastasis. ${ }^{3}$ There is increasing evidence that measures of the systemic inflammatory response, such as neutrophil, lymphocyte, C-reactive protein (CRP), and the Glasgow Prognostic Score (GPS), have prognostic value in a variety of cancers. ${ }^{4}$

The platelet-lymphocyte ratio (PLR), defined as the absolute platelet count divided by the absolute lymphocyte count, has gained a lot of interest in recent years. Published data suggested that elevated PLR was an important prognostic factor in esophageal cancer, ${ }^{5}$ gastric cancer, ${ }^{6}$ renal cell cancer, ${ }^{7}$ and malignant pleural mesothelioma. ${ }^{8}$ However, due to the inconsistent results, the prognostic value of PLR in NSCLC remains unclear. ${ }^{9-15}$ In this study, therefore, we performed a systematic review and meta-analysis including the most recent studies, aimed at achieving a relatively comprehensive and accurate conclusion concerning the prognostic significance of PLR in patients with NSCLC.

\section{Materials and methods}

\section{Literature search}

This meta-analysis was executed in accordance with the Preferred Reporting Items for Systematic Reviews and Meta-Analyses (PRISMA) guidelines. ${ }^{16}$ A systematic literature search was conducted by using PubMed, EMBASE, and Web of Science databases to assess the prognostic value of PLR in patients with NSCLC. The search strategy was based on combinations of the following search terms: ("platelet-to-lymphocyte ratio" or "plateletlymphocyte ratio" or "platelet lymphocyte ratio" or "PLR") and ("lung cancer" or "lung carcinoma" or "non-small cell lung cancer" or "non small cell lung cancer" or "NSCLC"). The last search was updated on May 20, 2015. Only human research was included in our meta-analysis. Moreover, the references in the identified articles were also retrieved for further relevant studies.

\section{Inclusion/exclusion criteria}

Studies were eligible for inclusion in this meta-analysis if they met the following criteria: 1) patients with NSCLC confirmed by pathology; 2) the PLR was measured before treatment; 3) investigated the relationship between PLR and prognosis (overall survival [OS], and disease-free survival [DFS]); 4) reported a hazard ratio (HR) and 95\% confidence intervals (CIs) or the data sufficient to estimate the HR and 95\% CIs; 5) to be published as full texts without language limits. The exclusion criteria were 1) abstracts, case reports, reviews, letters, editorials, and conference presentations;
2 ) insufficient data to extract or estimate the HRs and the $95 \%$ CIs.

\section{Data extraction}

The two primary investigators (Qiang and Guo) independently evaluated and extracted data from each study. All studies were double-checked and disagreements were resolved by discussion and consensus. Information extracted included the following: 1) publication details, including author, publication year, country, and study period; 2) characteristics of the studied population, including sample size, stage of disease, and PLR cutoff value; 3) HRs and 95\% CIs for the association between PLR and prognosis (OS and DFS). If several estimates were presented in the same article, we selected the most powerful one (multivariate analysis was superior to univariate analysis).

\section{Statistical analysis}

Pooled HRs and their 95\% CIs from each study were used to evaluate the relationship between PLR and prognosis. The heterogeneity of pooled results was analyzed by using Cochran's $Q$-test and Higgins' $I^{2}$ statistics. A significant heterogeneity was defined as $Q$-test $P$-value $<0.10$ or $I^{2}>50 \% .{ }^{17}$ When no significant heterogeneity existed among studies, the fixed-effects (Mantel-Haenszel method) model was used to generate the pooled HRs and 95\% CIs. Otherwise, the random-effects (DerSimonian-Laird method) model was applied. A combined HR $>1$ indicated a worse survival, and it was considered statistically significant if the 95\% CI did not overlap with the null value of one. Sensitivity analyses were performed to evaluate the stability of the results. Publication bias of literatures was assessed using funnel plot. All statistical tests were two sided, and the significance level was set at 5\%. All analyses were carried out with the statistical software Stata, version 12.0 (Stata corporation, College Station, TX, USA).

\section{Results}

The flow diagram of literature retrieval procedure is shown in Figure 1. The initial search strategies retrieved a total of 214 studies. After excluding duplicate studies and screening the titles or abstracts, ten full-text articles concerning PLR and the prognosis of NSCLC were further evaluated. Three of them were eliminated because they did not provide specific data for survival. Therefore, according to the search strategies, a total of seven eligible studies were included in our meta-analysis finally. Three studies were performed in the People's Republic of China, two were performed in Turkey, 


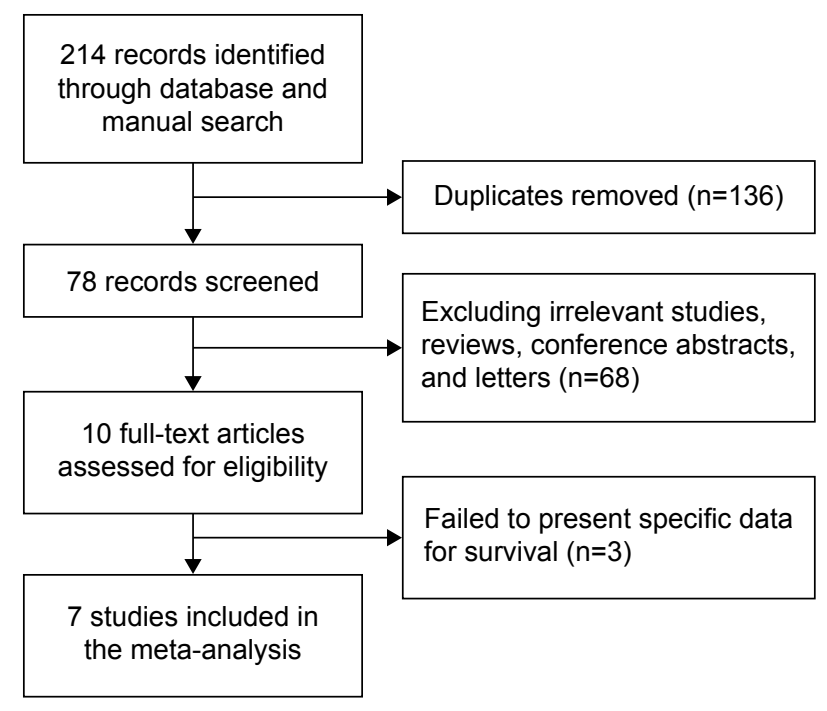

Figure I Flow diagram of study selection for the meta-analysis.

one in the UK, and one in Mexico, respectively. Two studies (Pinato et $\mathrm{al}^{12}$; Zhang et $\mathrm{al}^{13}$ ) included operable early stage cases (stage I-II or I-IIIA), four studies (Sánchez-Lara et al ${ }^{9}$; Liu et $\mathrm{a}^{10}{ }^{10}$, Unal et $\mathrm{al}^{11}$; Wu et a ${ }^{15}$ ) only included inoperable late-stage cases (stage III-IV), and one study (Kos et al ${ }^{14}$ ) included all stage cases (stage I-IV). A total of 1,554 patients (1,040 males, 514 females) with NSCLC were included in this meta-analysis. The number of patients in each study ranged from 94 to 400 . The cutoff value of PLR used in each study was not consistent, ranging from 119.5 to 300.0, with a median of 171 . The main characteristics of included studies are summarized in Table 1.

All these seven studies presented data on PLR and OS, but only three studies reported data on PLR and DFS. Our pooled results demonstrated that high PLR was significantly associated with poor OS (HR: $1.60,95 \% \mathrm{CI}: 1.34-1.90, I^{2}=22.3 \%$, $\left.P_{\text {heterogeneity }}=0.259\right)$ (Figure 2$)$ and DFS (HR: $1.38,95 \% \mathrm{CI}$ : $\left.1.11-1.73, P^{2}=0 \%, P_{\text {heterogeneity }}=0.482\right)$ (Figure 3$)$. There was no significant heterogeneity between these studies.

In a further investigation, subgroup analyses were performed with confounders such as ethnicity, sample size, and cutoff value (Figure 4). Subgroup analyses by ethnicity indicated that high PLR predicted poor prognosis for patients both in Caucasian populations (HR $=1.56,95 \%$ CI: $1.19-2.04, P=0.001)$ and in Asian cases $(\mathrm{HR}=1.62$, 95\% CI: 1.29-2.04, $P<0.001$ ) (Figure 4A). When studies were stratified by sample size, we found the combined HRs were $1.56,95 \%$ CI: $1.18-2.06$ for studies with less than 200 cases and HR 1.62, 95\% CI: 1.30-2.03 for studies with more than 200 cases (Figure 4B). This result demonstrated that high PLR remained to be an unfavorable prognostic

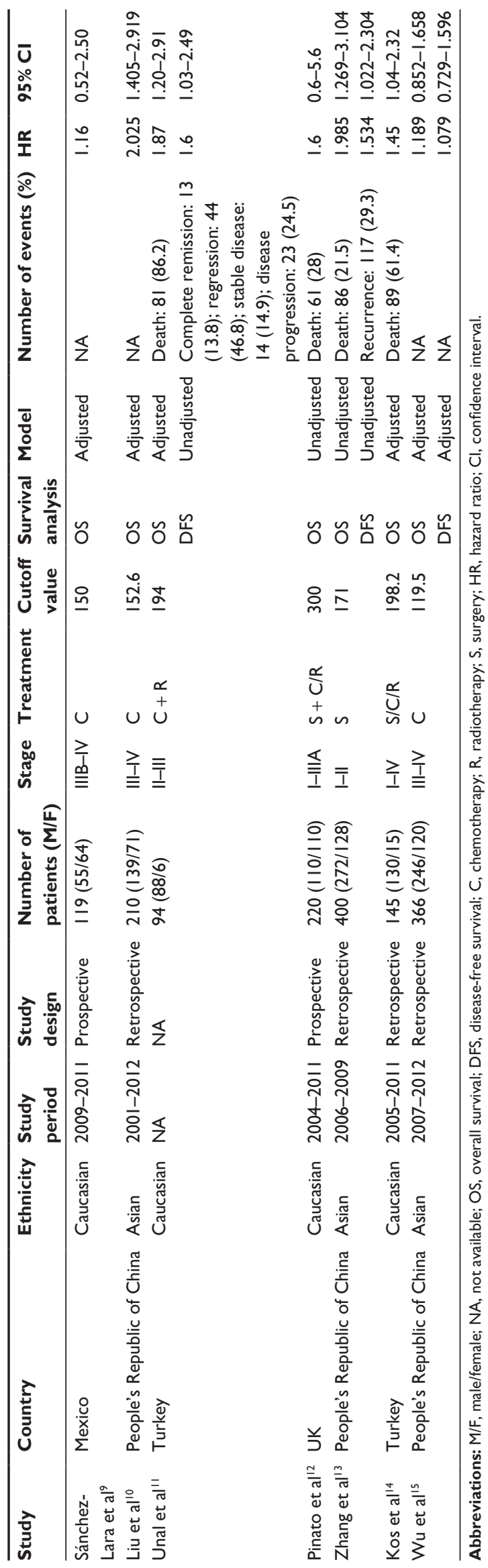




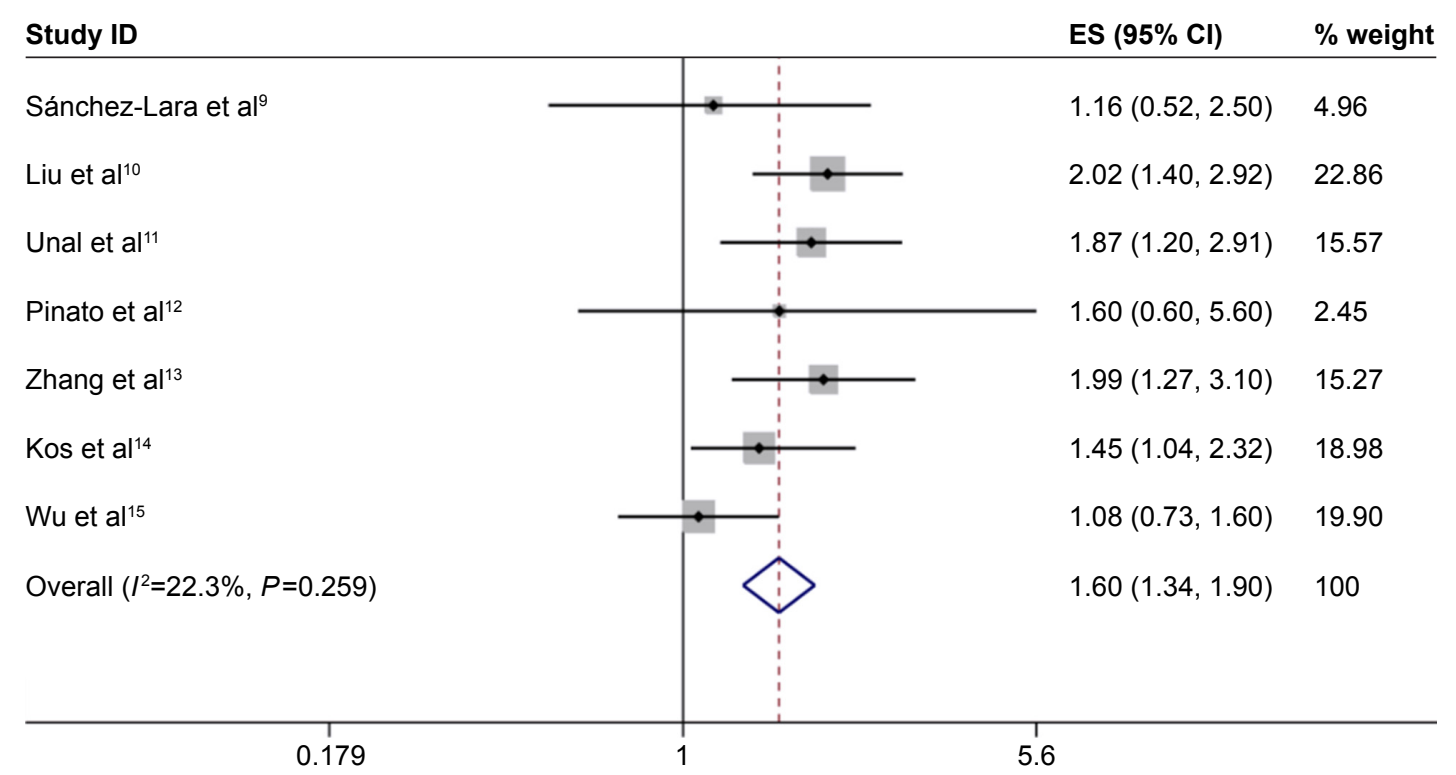

Figure 2 Forest plot of the association between PLR and OS in NSCLC.

Abbreviations: $\mathrm{ES}$, effect size; $\mathrm{Cl}$, confidence interval; PLR, platelet-to-lymphocyte ratio; OS, overall survival; NSCLC, non-small cell lung cancer.

factor regardless of sample size. With stratification by cutoff value $\leq 171$ and cutoff value $>171$, the data showed that the pooled HR was 1.58 (95\% CI: $1.27-1.97)$ and 1.62 (95\% CI: $1.22-2.17$ ), respectively (Figure $4 \mathrm{C}$ ). When studies were stratified by stage and treatment modality, we found the combined HRs were 1.56, 95\% CI: (1.25-1.94) for studies with inoperable late-stage cases and 1.93, 95\% CI: (1.27-2.92) for studies with operable early stage cases (Figure 4D). This result demonstrated that high PLR remained to be an unfavorable prognostic factor regardless of disease stage and treatment modality.

We conducted leave-one-out sensitivity analysis to check if any individual study affected the results. We found that removing any single study in turn did not significantly affect the pooled HRs in the present meta-analysis (Figure 5). This result indicates that the present meta-analysis was proven to have good reliability.

The Begg's funnel plot was performed to evaluate publication bias of the included studies. As shown in Figure 6, the funnel plot was symmetric. There was no significant publication bias in our study.

\section{Discussion}

To the best of our knowledge, this is the first meta-analysis to investigate the prognostic value of PLR in patients with NSCLC. In our meta-analysis, the pooled results demonstrated

\begin{tabular}{|c|c|c|}
\hline Study ID & ES $(95 \% \mathrm{Cl})$ & $\%$ weight \\
\hline Unal et al ${ }^{11}$ & $1.60(1.03,2.49)$ & 25.40 \\
\hline Zhang et $\mathrm{al}^{13}$ & $1.53(1.02,2.30)$ & 29.95 \\
\hline Wu et al ${ }^{15}$ & $1.19(0.85,1.66)$ & 44.65 \\
\hline Overall $\left(I^{2}=0.0 \%, P=0.482\right)$ & $1.38(1.11,1.73)$ & 100 \\
\hline
\end{tabular}

Figure 3 Forest plot of the association between PLR and DFS in NSCLC.

Abbreviations: $\mathrm{ES}$, effect size; $\mathrm{Cl}$, confidence interval; PLR, platelet-to-lymphocyte ratio; DFS, disease-free survival; NSCLC, non-small cell lung cancer. 


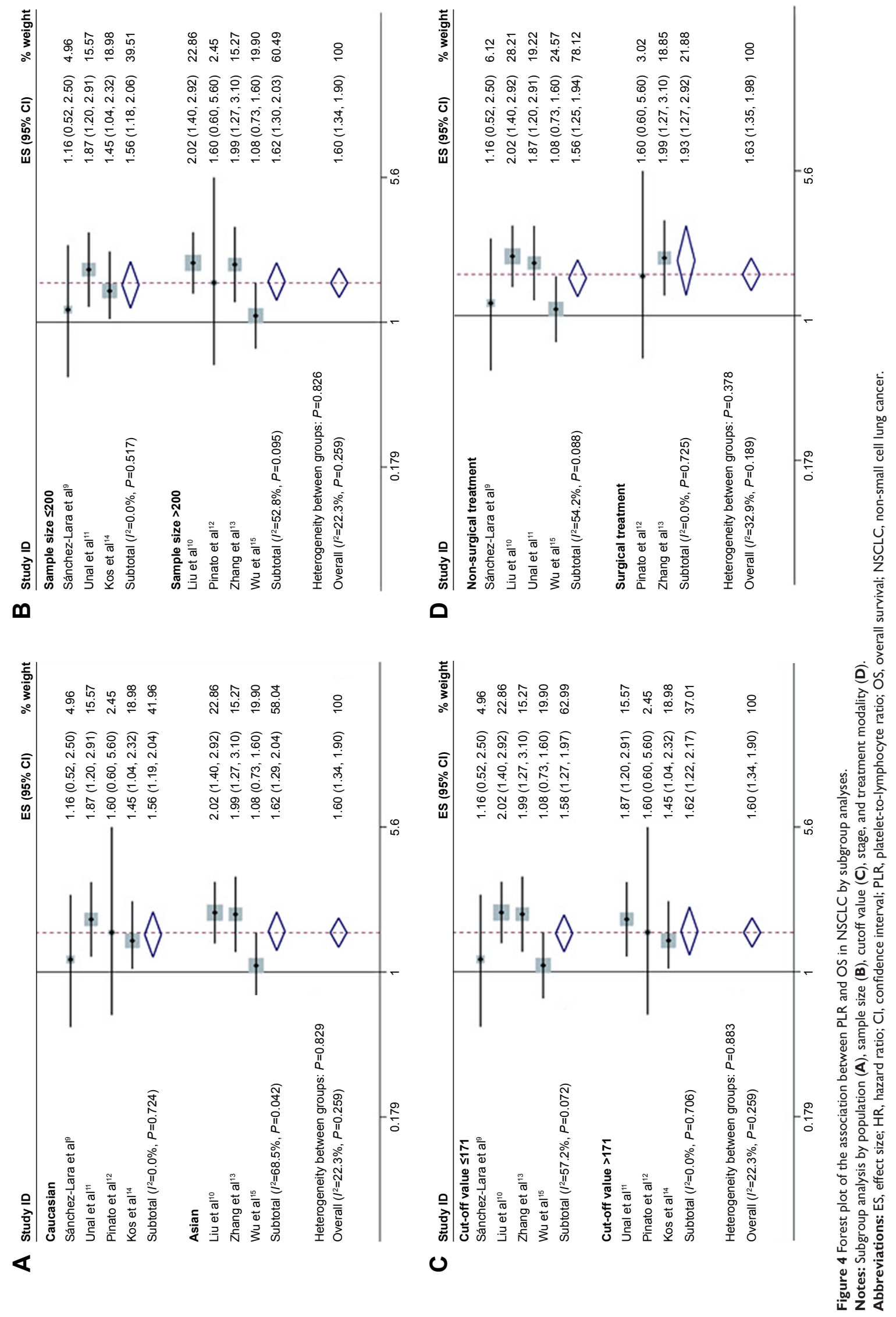




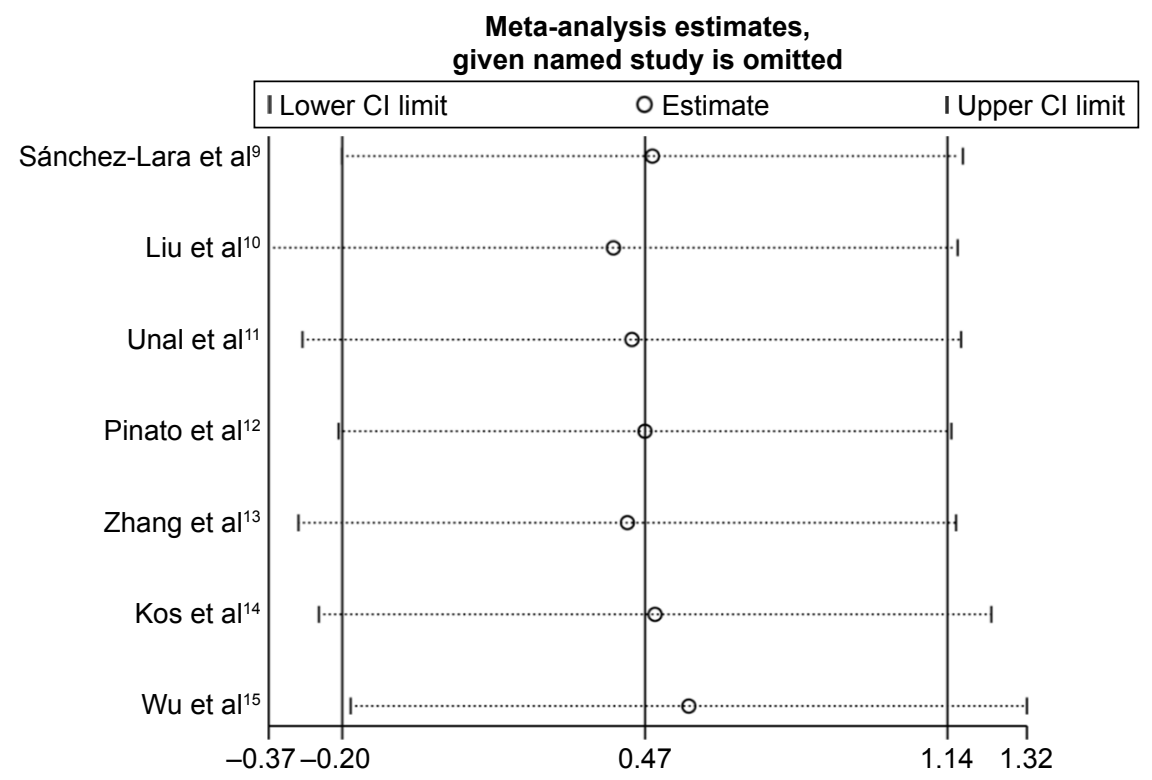

Figure 5 Sensitivity analysis on the relationship between PLR and OS in NSCLC.

Abbreviations: $\mathrm{Cl}$, confidence interval; PLR, platelet-to-lymphocyte ratio; OS, overall survival; NSCLC, non-small cell lung cancer.

that high PLR was associated with poor OS (HR: 1.60, 95\% CI: $\left.1.34-1.90, I^{2}=22.3 \%, P_{\text {heterogeneity }}=0.259\right)$ and DFS (HR: $\left.1.38,95 \% \mathrm{CI}: 1.11-1.73, I^{2}=0 \%, P_{\text {heterogeneity }}=0.482\right)$ in patients with NSCLC. We performed further subgroup analyses to evaluate the association between PLR and OS in the included studies. When the patients were segregated according to ethnicity and sample size, high PLR was also significantly correlated with OS. These results, on the other hand, indicated that the high levels of PLR could significantly predict the poor OS in patients with NSCLC regardless of ethnicity and sample size. In addition, when stratified by cutoff value, the results were also significant no matter what cutoff value was used.

Over the past few decades, a number of prognostic factors for NSCLC have been identified, including TNM stage,

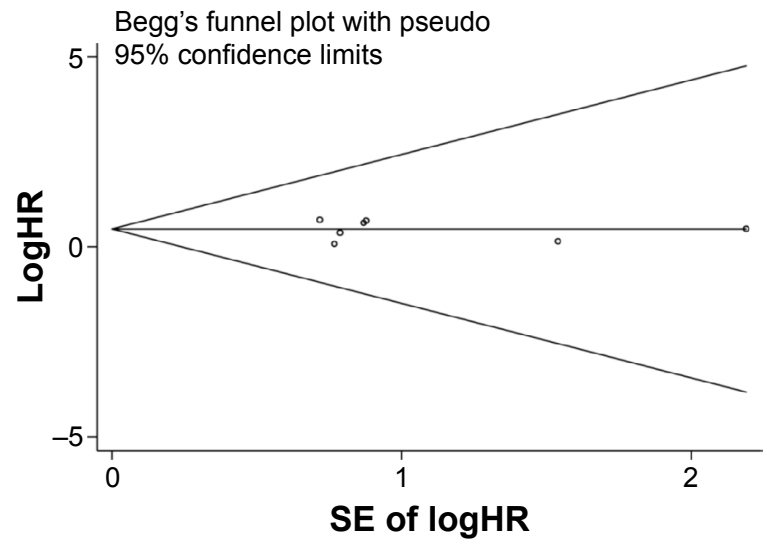

Figure 6 Funnel plot for detecting publication bias. Abbreviations: HR, hazard ratio; SE, standard error. genetic factors, and performance status. Recently, there is increasing evidence that a systemic inflammatory response is of prognostic value in various types of cancers. Several inflammatory biomarkers such as PLR, NLR, CRP, and the modified Glasgow Prognostic Score (mGPS) are associated with prognosis of cancer patients. ${ }^{18}$ Pretreatment PLR is inversely related to prognosis in numerous cancers. However, its role in NSCLC is still unclear. The results of our study support the view that a high pretreatment PLR is a poor prognostic factor in NSCLC. Furthermore, the sensitivity analysis found that no single study could affect the pooled HRs in our meta-analysis. There was no publication bias in our study. These findings indicated that our meta-analysis was stable and reliable.

However, the mechanism underlying the association of PLR with prognosis of NSCLC is still incompletely understood. Platelets play an important role in cancer progression. Previously published studies have proposed that thrombocytosis is an independent unfavorable prognostic factor in patients with NSCLC. ${ }^{19}$ Platelet (PLT) aggregation promotes the adhesion and encapsulation of circulating tumor cells. This enhances the ability of tumor cells to escape from antitumor immunity. ${ }^{20}$ Furthermore, activated PLT release vascular endothelial growth factor and numerous cytokines, which stimulate tumor growth by increasing angiogenesis. ${ }^{21-23}$ In addition, increased PLT aggregation supposed to be involved in the development of hematogenous metastases by promoting microcirculatory adhesion and colonization 
of tumor cells. ${ }^{24}$ On the other hand, lymphocytes are major compartments of the immune system, which play a key role in defending against cancers. Zhang et $\mathrm{al}^{25}$ reported elevated peripheral lymphocytes to be a favorable prognostic factor. Similarly Kobayashi et $\mathrm{al}^{26}$ reported that a low lymphocyte count was an independent unfavorable prognostic factor in patients with resected node negative NSCLC. Thus, lymphocytopenia reflects impaired lymphocyte-mediated antitumor reaction. As a combination of circulating platelet and lymphocyte counts, an elevated PLR represent both thrombocytosis and lymphocytopenia, thereby contributing to aggressive cancer progression and poor survival. However, further investigation is needed to explain the exact mechanism.

There were some limitations in our study. First, the number of included studies was limited. Second, due to lack of relevant prospective studies, most of the included studies were retrospective and single institution case series. Third, because of the lack of appropriate data, the correlation between PLR and other clinicopathological parameters was not analyzed.

In conclusion, the current meta-analysis revealed that high level of PLR was significantly associated with poor OS and DFS in patients with NSCLC. As PLR is readily available and cost-effective, we propose that PLR should be used as a prognostic biomarker to improve selection of treatment modalities for individual NSCLC patients. However, the clinical utility of the PLR needs to be validated by more well-designed and large-scale investigations in the future.

\section{Disclosure}

The authors report no conflicts of interest in this work.

\section{References}

1. Powell CA, Halmos B, Nana-Sinkam SP. Update in lung cancer and mesothelioma 2012. Am J Respir Crit Care Med. 2013;188(2):157-166.

2. Balkwill F, Mantovani A. Inflammation and cancer: back to Virchow? Lancet. 2001;357(9255):539-545.

3. Shi L, Wang L, Hou J, et al. Targeting roles of inflammatory microenvironment in lung cancer and metastasis. Cancer Metastasis Rev. 2015;34(2):319-331.

4. McMillan DC. The systemic inflammation-based Glasgow Prognostic Score: a decade of experience in patients with cancer. Cancer Treat Rev. 2013;39(5):534-540.

5. Dutta S, Crumley AB, Fullarton GM, Horgan PG, McMillan DC. Comparison of the prognostic value of tumour- and patient-related factors in patients undergoing potentially curative resection of oesophageal cancer. World J Surg. 2011;35(8):1861-1866.

6. Dutta S, Crumley AB, Fullarton GM, Horgan PG, McMillan DC. Comparison of the prognostic value of tumour and patient related factors in patients undergoing potentially curative resection of gastric cancer. Am J Surg. 2012;204(3):294-299.
7. Fox P, Hudson M, Brown C, et al. Markers of systemic inflammation predict survival in patients with advanced renal cell cancer. Br J Cancer. 2013;109(1):147-153.

8. Pinato DJ, Mauri FA, Ramakrishnan R, Wahab L, Lloyd T, Sharma R. Inflammation-based prognostic indices in malignant pleural mesothelioma. J Thorac Oncol. 2012;7(3):587-594.

9. Sánchez-Lara K, Turcott JG, Juárez E, et al. Association of nutrition parameters including bioelectrical impedance and systemic inflammatory response with quality of life and prognosis in patients with advanced non-small-cell lung cancer: a prospective study. Nutr Cancer. 2012;64(4):526-534.

10. Liu H, Wu Y, Wang Z, et al. Pretreatment platelet-to-lymphocyte ratio (PLR) as a predictor of response to first-line platinum-based chemotherapy and prognosis for patients with non-small cell lung cancer. J Thorac Dis. 2013;5(6):783-789.

11. Unal D, Eroglu C, Kurtul N, Oguz A, Tasdemir A. Are neutrophil/ lymphocyte and platelet/lymphocyte rates in patients with non-small cell lung cancer associated with treatment response and prognosis? Asian Pac J Cancer Prev. 2013;14(9):5237-5242.

12. Pinato DJ, Shiner RJ, Seckl MJ, Stebbing J, Sharma R, Mauri FA. Prognostic performance of inflammation-based prognostic indices in primary operable non-small cell lung cancer. Br J Cancer. 2014; 110(8):1930-1935.

13. Zhang T, Jiang Y, Qu X, Shen H, Liu Q, Du J. Evaluation of preoperative hematologic markers as prognostic factors and establishment of novel risk stratification in resected $\mathrm{pN} 0$ non-small-cell lung cancer. PLoS One. 2014;9(10):e111494.

14. Kos M, Hocazade C, Kos FT, et al. Prognostic role of pretreatment platelet/lymphocyte ratio in patients with non-small cell lung cancer. Wien Klin Wochenschr. Epub February 27, 2015.

15. Wu G, Yao Y, Bai C, et al. Combination of platelet to lymphocyte ratio and neutrophil to lymphocyte ratio is a useful prognostic factor in advanced non-small cell lung cancer patients. Thorac Cancer. 2015;6(3):275-287.

16. Moher D, Liberati A, Tetzlaff J, Altman DG; PRISMA Group. Preferred reporting items for systematic reviews and meta-analyses: the PRISMA statement. BMJ. 2009;339:b2535.

17. Higgins JP, Thompson SG, Deeks JJ, Altman DG. Measuring inconsistency in meta-analyses. BMJ. 2003;327(7414):557-560.

18. Roxburgh CS, McMillan DC. Cancer and systemic inflammation: treat the tumour and treat the host. Br J Cancer. 2014;110(6):1409-1412.

19. Tomita M, Shimizu T, Hara M, Ayabe T, Onitsuka T. Prognostic impact of thrombocytosis in resectable non-small cell lung cancer. Interact Cardiovasc Thorac Surg. 2008;7(4):613-615.

20. Jurasz P, Alonso-Escolano D, Radomski MW. Platelet - cancer interactions: mechanisms and pharmacology of tumour cell-induced platelet aggregation. Br J Pharmacol. 2004;143(7):819-826.

21. Bambace NM, Holmes CE. The platelet contribution to cancer progression. J Thromb Haemost. 2011;9(2):237-249.

22. Sierko E, Wojtukiewicz MZ. Platelets and angiogenesis in malignancy. Semin Thromb Hemost. 2004;30(1):95-108.

23. Menter DG, Tucker SC, Kopetz S, Sood AK, Crissman JD, Honn KV. Platelets and cancer: a casual or causal relationship: revisited. Cancer Metastasis Rev. 2014;33(1):231-269.

24. Morimoto K, Satoh-Yamaguchi K, Hamaguchi A, et al. Interaction of cancer cells with platelets mediated by Necl-5/poliovirus receptor enhances cancer cell metastasis to the lungs. Oncogene. 2008;27(3):264-273.

25. Zhang J, Huang SH, Li H, et al. Preoperative lymphocyte count is a favorable prognostic factor of disease-free survival in non-small-cell lung cancer. Med Oncol. 2013;30(1):352.

26. Kobayashi N, Usui S, Kikuchi S, et al. Preoperative lymphocyte count is an independent prognostic factor in node-negative non-small cell lung cancer. Lung Cancer. 2012;75(2):223-227. 


\section{Publish your work in this journal}

OncoTargets and Therapy is an international, peer-reviewed, open access journal focusing on the pathological basis of all cancers, potential targets for therapy and treatment protocols employed to improve the management of cancer patients. The journal also focuses on the impact of management programs and new therapeutic agents and protocols on

patient perspectives such as quality of life, adherence and satisfaction. The manuscript management system is completely online and includes a very quick and fair peer-review system, which is all easy to use. Visit http://www.dovepress.com/testimonials.php to read real quotes from published authors.

Submit your manuscript here: http://www.dovepress.com/oncotargets-and-therapy-journal 